\title{
A new approach to tuberculosis notification
}

\author{
N J SHANKS, A LAMBOURNE, R A KUHAYMI, M HUMPHRiES, AND J R A \\ SANFORD
}

From the Tuberculosis Notification Office, Riyadh Military Hospital, Riyadh, Kingdom of Saudi Arabia

SUMMARY A tuberculosis notification system was initiated which used several sources - the doctor, the diagnostic services of bacteriology, histopathology and radiology, and the pharmacy. The results of six months' notifications have shown that the pharmacy was by far the most complete source ( $74 \%$ of all new cases); doctors notified only $21 \%$ of all new cases. The combination of both doctors and the pharmacy was the most complete pair, notifying $85 \%$ of all new cases. It is suggested that consideration should be given to the use of pharmacies as a source of notification both in other settings and for other diseases.

Tuberculosis remains one of the most important infectious diseases in the world. It is estimated that each year about 10 million people still develop tuberculosis and at least 3 million die from the disease. ${ }^{1}$ Estimates of overall incidence can be made from surveys; the collection of data on individual cases is usually by a disease notification system. ${ }^{2}$ Although in most countries physicians have a statutory duty to notify new cases of tuberculosis, it is well recognised that physicians are unreliable in their notifications of this disease. ${ }^{3}$ Other sources of notification have been used in some countries with better effect. ${ }^{34}$

It is documented that there are many defects in most current systems of recording and reporting tuberculosis, ${ }^{35}$ and the importance of a well planned and standardised approach to all aspects of data processing from collection to analysis and prevention is regularly stressed. ${ }^{67}$

The results are presented of a study to ascertain the completeness of tuberculosis notifications which arose from several often unused sources.

\section{Method}

On 1 January 1983 a tuberculosis notification system was introduced in the Riyadh and Al Kharj Armed Forces Hospitals Programme. ${ }^{8}$

To encourage the notification of cases, all physicians were informed of the new system in mid-December 1982. Each received a personal letter accompanied by a sample notification form and instructions on how to complete the form.
Notification forms were made available in all wards, outpatient departments, and the primary care departments of both hospitals. In addition, the system was advertised on posters throughout both hospitals.

To obtain as complete a notification system as possible, four other sources were also used: the bacteriology, histopathology, radiology, and pharmacy departments of the hospitals.

The x-ray department began a log-book in which all suspected cases were to be recorded. For the bacteriology department the results were already part of their record system. For histology, no scheme was begun, and the data had to be collected by one of the authors (NS) manually reviewing all the individual test forms of the histology department.

Finally, the pharmacy department was asked to record on a predesigned form all patients who were receiving prescriptions for the anti-tuberculosis drugs.

The data from all these sources have been collected and collated monthly and the data for the first six months of 1983 are presented in this paper.

\section{Results}

The results are shown in Tables 1 to 3 . In all tables the sources have been listed in rank order of the numbers of notifications that originated from them.

Table 1 shows the numbers of notifications that would have been received had each of the five sources been used alone. Using a single source, the pharmacy was the clear leader, with $74 \%$ of all cases 
notified. Doctors notified only $21 \%$ of the total cases, bacteriology $13 \%$, and both radiology and histology less than $10 \%$.

Table 1 Total cases notified using single sources

\begin{tabular}{lrr}
\hline Source & No. & $\%^{*}$ \\
\hline Pharmacy & 331 & 74 \\
Doctors & 92 & 21 \\
Bacteriology & 56 & 13 \\
Radiology & 34 & 8 \\
Histology & 26 & 6 \\
\hline
\end{tabular}

*Percentages of the total 446 notifications.

Table 2 shows the notifications that would have arisen had a pair of sources been used. Pharmacy's pairs rank highest. Pharmacy and doctors together notified 377 cases $(85 \%$ of the total); this was 46 (11\% of the total) higher than originated from the pharmacy alone. Pharmacy and bacteriology together notified 368 cases ( $83 \%$ of the total), only nine fewer than the combination of pharmacy and doctors-even though bacteriology notified only 56 cases. This was due to most of the cases notified by bacteriology being different from those notified by pharmacy. Similarly, pharmacy and radiology notified 359 cases $(80 \%)$ and pharmacy and histology $351(79 \%)$. The other pairs of sources all provided less than one-third of all cases notified.

Table 2 Total cases notified using pairs of sources

\begin{tabular}{lcc}
\hline Combination & No. & $\%^{*}$ \\
\hline Pharmacy and & 377 & 85 \\
Doctors & 368 & 83 \\
Bacteriology & 359 & 80 \\
Radiology & 351 & 79 \\
Histology & & \\
$\begin{array}{l}\text { Doctors and } \\
\text { Bacteriology }\end{array}$ & 142 & 32 \\
Radiology & 123 & 28 \\
Histology & 116 & 26 \\
Bacteriology and & & \\
Radiology & & \\
Histology & 90 & 20 \\
Radiology and & 78 & 17 \\
Histology & & \\
\hline
\end{tabular}

*Percentages of the total 446 notifications.

The reason for no single source or pair of sources accounting for more than $85 \%$ of all notifications is shown in Table 3 . This table shows the notifications unique to each source, that is, notified by that source and no other. More than half of all notifications $(57 \%)$ were unique to pharmacy, $8 \%$ to doctors, $6 \%$ to bacteriology, $5 \%$ to radiology, and $3 \%$ to histology. The reasons for the latter three diagnostic sources' notified cases being missed by both the doctor and pharmacy are being investigated.

Table 3 Notifications unique to each source

\begin{tabular}{lrc}
\hline Unique Source & No. & $\% *$ \\
\hline Pharmacy & 254 & 57 \\
Doctor & 35 & 8 \\
Bacteriology & 27 & 6 \\
Radiology & 24 & 5 \\
Histology & 13 & 3 \\
\hline
\end{tabular}

*Percentages of the total 446 notifications.

Table 4 Sources of the 115 cases not notified by the pharmacy

\begin{tabular}{lcc}
\hline Source & No. & $\%$ \\
\hline Doctor only & 35 & 30 \\
Bacteriology only & 27 & 23 \\
Radiology only & 24 & 21 \\
Histology only & 13 & 11 \\
Combination of above & 16 & 14 \\
Total & 115 & 100 \\
\hline
\end{tabular}

\section{Discussion}

This is the first time that the notifications of a single disease from five different sources have beerf compared. Indeed, it is believed to be the first time? that the use of pharmacy as a source in the disease notification system has ever been considered. The results clearly show that the pharmacy provides the most complete set of tuberculosis notifications.

Doctors are reputed to regard extraclerical duties, such as the completion of notification forms, with distaste-even if given a financial incentive. ${ }^{3}$ Although nurses and clerical staff have been used to complete the forms in some countries ${ }^{3}$ they are still dependent on a doctor initiating the notification and, for legal purposes, authorising it. This study supports these previous observations, only $21 \%$ of all cases being notified by doctors. While some cases not notified by doctors may have been missed by genuine administrative errors, such as missing laboratory results, unavailability of records, etc, such errors could explain only a small proportion of the large number of non-notified cases (354 out of 446).

The use of the diagnostic services of bacteriology, histopathology, and radiology has its limitations in each case. While all suspect cases may have bacteriological tests performed, only a small proportion can be expected to yield positive results. 
Conversely, pulmonary tuberculosis, which constitutes the largest proportion of all cases, does not usually require histopathological confirmation.

Likewise, radiography is not applicable to the diagnosis of lymphadenitis, which is the commonest form of non-pulmonary tuberculosis in Saudi Arabia. $^{9}$

Although the biggest additional contribution was from doctors, bacteriology was nearly as good an additional source, and fuller laboratory reporting might help considerably with diagnosed cases under private treatment or not starting the prescribed course.

However, once diagnosed-by whatever means-a patient considered to have tuberculosis will undergo a prolonged period of chemotherapy, the drugs for which are restricted to mycobacteria. It is for this reason that the pharmacy was by far the most complete source of notification. Of the 331 cases notified by the pharmacy, only seven were found to be cases of other diseases-four were cases of leprosy and three were found not to have been prescribed the anti-tuberculosis drugs. Although the cases of leprosy may be considered as false notifications, they also need surveillance, long term follow-up, and contact tracing. In fact, in some countries, the tuberculosis and leprosy control programmes have been united. ${ }^{3}$

However, there were still 115 of the 446 cases not notified by the hospitals' pharmacy departments. The sources of the 115 cases not notified by the pharmacy are clearly tabulated.

There were two main reasons:

(a) Some issues of the specified drugs were not being notified by the hospital's pharmacies; and

(b) Some cases diagnosed at the hospitals were using private pharmacies for their drugs, or never began the prescribed course of treatment.

The pharmacy notification itself could be improved by ensuring that each pharmacist is fully aware of the importance of recording and reporting every anti-tuberculosis drug prescriber.

As this study was purely a voluntary system some pharmacists may have been cursory in their approach to the notifications. However, their voluntary response has been clearly laudable. Nevertheless, the rate of notification could be almost complete were it to be introduced as a mandatory system, as in the case of dangerous drugs.

The use of a pharmacy for disease notification has application to other diseases with disease-specific treatment regimes. Important examples are leprosy, malaria and schistosomiasis. While it is recognised that their drugs may be used for other conditions, the numbers of 'false positive' notifications for the disease under surveillance would be relatively small.
The principal advantages of using a pharmacy as a source of notifications are:

1 Clerical work is a routine part of a pharmacy's work, and one extra duty will not be considered a burden.

2 As found in other studies, ${ }^{3}$ the involvement of non-medical staff in disease surveillance is often considered to enhance their other duties. They see themselves contributing more directly in a disease control programme, and, provided they are kept informed of the practical use being made of their work, they are both diligent and reliable.

While the environment in which this study was carried out had the advantage of on-site pharmacies, the use of all pharmacies for the notification of tuberculosis is clearly practical. It would be possible by:

1 permitting only selected pharmacies to stock anti-tuberculosis drugs;

2 making the participation in the notification system a prerequisite of a pharmacy dispensing such drugs;

3 providing a suitable financial incentive for either each individual notification or a weekly report of all prescriptions.

One or more of the above would provide as complete a notification system as is possible.

The relevance of pharmacy notification in other countries with more or less well developed health care programmes requires evaluation in each case by a pilot project as in the present study. Where similar results were obtained implementation of pharmacy notification could contribute greatly to the elimination of tuberculosis, as case follow-up and contact-tracing could, in most cases, identify the $15 \%$ missed by the notification system, including the foci that generate the outbreaks. The greatest value of using such a system would be in countries where pharmacies are government controlled or where the numbers that dispense anti-tuberculosis chemotherapy are limited. In developed countries, such as the United Kingdom, the logistics of the collection of data from the large number of pharmacies dispensing anti-tuberculosis drugs to a small number of tuberculosis patients would render this system less practical. Thus, the introduction of pharmacy notification could be a significant step towards the goal of total eradication of tuberculosis in the developing world.

Our sincere thanks to $\mathrm{Mr} \mathbf{R}$ Quested, chief pharmacist, and the RKH Pharmacy Department for their help and co-operation in this study. 


\section{References}

${ }^{1}$ World Health Organisation. Tuberculosis control. Technical Report Series No. 671; 1982: 10.

${ }^{2}$ World Health Organisation. World health statistics annual. Infectious diseases. 1981.

${ }^{3}$ Lambourne A. Notifiable disease information systems and their application to a mathematical model for the control of measles in the Caribbean. PhD thesis, Sheffield University, UK. 1983.

${ }^{4}$ Schaffner W, Scott HD, Rosenstein BJ, Byrne EB. Innovative communicable disease reporting. $H S M H A$ Health Reports 1971; 86: 41-.
${ }^{5}$ Marier R. The reporting of communicable diseases. $A m \mathrm{~J}$ Epidemiol 1987: 105: ,587-90.

${ }^{6}$ Lossing EG. Reporting of notifiable diseases. Can $J$ Public Health 1955: 4444-448.

${ }^{7}$ Swaroop S. Introduction to health statistics. London: Livingstone, 1960.

${ }^{8}$ Shanks NJ, Humphries M. A notification system for tuberculosis. Saudi Medical Journal 1983; 4: 1171-3.

${ }^{9}$ Shanks NJ, Khalifa I, Al Kalai D. Tuberculosis in Saudi Arabia. Saudi Medical Journal 1983; 4: 151-6. 